\title{
Short Wavelength Geodesic Acoustic Mode Excitation by Energetic Particles
}

\author{
Liu Chen ${ }^{1,2}$, Zhiyong Qiu ${ }^{1}$ and Fulvio Zonca ${ }^{3,1}$ \\ ${ }^{1}$ Institute for Fusion Theory and Simulation and Department of Physics, Zhejiang University, Hangzhou, P.R.C \\ ${ }^{2}$ Department of Physics and Astronomy, University of California, Irvine CA 92697-4575, U.S.A. \\ 3 ENEA, Fusion and Nuclear Safety Department, \\ C. R. Frascati, Via E. Fermi 45, 00044 Frascati (Roma), Italy
}

\begin{abstract}
Taking the collisionless damping of geodesic acoustic mode (GAM) as an example, the physics processes underlying wave particle resonances in the short wavelength limit are clarified. As illustrative application, GAM excitation by energetic particles in short wavelength limit is investigated assuming a single pitch angle slowing-down fast ion equilibrium distribution function. Conditions for this energetic particle-induced GAM (EGAM) to be unstable are discussed.
\end{abstract}

Recently, due to geodesic acoustic mode (GAM) [1] excitation by energetic particles (EPs) in the large drift orbit limit [2], there has been renewed interest in waveparticle resonances at short wavelength, which was firstly investigated in Ref. 3 for the collisionless damping of GAM, and presented later providing detailed derivation and physics interpretation [4]. The same approach was also applied to study quasi-linear transport of EPs by drift wave turbulence [5, 6]. However, the understanding of the underlying physics processes proposed in recent literature, e.g. [2], may yield to some mis-interpretation and inconsistency with the existing theoretical framework. In this brief communication, our aim is to clarify the underlying physics processes for wave-particle resonance in the short wavelength limit and, as illustrative application, investigate EP-induced GAM (EGAM) 7 [ 9 excitation by fast ions with large magnetic drift orbits.

To discuss the physics picture of wave-particle resonance in the short wavelength limit, we take electrostatic GAM collisionless damping originally discussed in [3, 4] as example. For the clarity of discussion, we assume small but finite electron temperature, i.e., $\tau \equiv T_{e} / T_{i} \ll 1$ such that $\left|\widetilde{\delta \phi}_{G} / \overline{\delta \phi}_{G}\right| \sim \tau k_{r} \rho_{t i} \ll 1$ while one still has $\left|\omega_{t r, e}\right| \gg\left|\omega_{G}\right|$. Here, $\widetilde{\delta \phi}_{G}$ and $\overline{\delta \phi}_{G}$ are respectively the $m \neq 0$ and $m=0$ components of the perturbed scalar potential; $\omega_{t r} \equiv v_{\|} /\left(q R_{0}\right)$ is the transit frequency, $k_{r}$ is the radial wavenumber and $\rho_{t i}$ is the ion Larmor radius at thermal velocity. In this limit, consistent with the short wavelength assumption of interest here, the perturbed electron response (distribution function) to GAM is $\delta f_{e}=0$, and the GAM dispersion relation can be derived from the quasi-neutrality condition:

$$
\sum_{s}\left\langle\delta f_{s}\right\rangle=0
$$

Here, $\langle\cdots\rangle$ denotes velocity space integration, subscript $s$ denotes different ions species and, thus, equation (11) can also be applied to study EGAM excitation by EPs. $\delta f_{s}$ can be expressed as $\delta f_{s}=e \partial_{E} F_{0} \delta \phi / m+$ $\exp \left[i\left(m_{i} c\right) /\left(e B^{2}\right) \mathbf{k} \times \mathbf{B} \cdot \mathbf{v}\right] \delta H$, and the nonadiabatic response can be derived from the following linear gyrokinetic equation [10, 11]:

$$
\left(-i \omega+\omega_{t r} \partial_{\theta}+i \omega_{d}\right) \delta H_{k}=i \omega\left(e / m_{i}\right) \partial_{E} F_{0} J_{k} \overline{\delta \phi}_{G}
$$

with $\omega_{d}=\hat{\omega}_{d} \sin \theta=k_{r} \rho_{t i} v_{t i}\left(v_{\perp}^{2} / 2+v_{\|}^{2}\right) /\left(v_{t i}^{2} R_{0}\right) \sin \theta$ being the magnetic drift frequency due to geodesic curvature, $J_{k} \equiv J_{0}\left(k_{r} \rho_{t i}\right)$ with $J_{0}$ being Bessel function of zero-order accounting for finite Larmor radius effects, $v_{t i} \equiv \sqrt{2 T_{i} / m_{i}}$ being the ion thermal velocity, $E=v^{2} / 2$ and other notations are stardard.

Noting that $\omega_{G} \simeq v_{t i} / R_{0} \sim q \omega_{t r, i} \gg \omega_{b, i} \simeq \sqrt{\epsilon} \omega_{t r, i}$, and assuming well circulating particles in the large aspect ratio limit, equation (2) can be solved and yields, for $v_{\|}>0$,

$$
\delta H_{s}=\frac{\omega}{\omega_{t r}} \hat{S} e^{-\psi(\theta)} \int_{-\infty}^{\theta} e^{\psi\left(\theta^{\prime}\right)} d \theta^{\prime}
$$

Here, $\hat{S} \equiv-i\left(e / m_{i}\right) \partial_{E} F_{0} J_{G} \overline{\delta \phi}_{G}, \psi(\theta) \equiv-i(\omega \theta+$ $\left.\hat{\omega}_{d} \cos \theta\right) / \omega_{t r}, \omega_{b}$ is the bounce frequency of trapped particles, and $\epsilon \equiv r / R_{0}$ is the inverse aspect ratio. Similar expression can also be obtained for $v_{\|}<0$.

Noting that

$$
e^{i \hat{\Lambda} \cos \theta}=\sum_{l} i^{l} J_{l}(\hat{\Lambda}) e^{i l \theta},
$$

the integration in $\theta^{\prime}$ in equation (3) can be carried out by transforming into transit harmonics, and one obtains

$$
\delta H_{s}=i \omega \hat{S} \sum_{p} i^{p} J_{p}(\hat{\Lambda}) e^{i p \theta} \sum_{l} \frac{(-i)^{l} J_{l}(\hat{\Lambda}) e^{i l \theta}}{\omega-l \omega_{t r}} .
$$

Here, $\hat{\Lambda} \equiv \hat{\omega}_{d} / \omega_{t r}$ and $\exp (-i \hat{\Lambda} \cos \theta)$ is the "pullback" (coordinate transformation) from drift orbit center to particle guiding center coordinates. The resonance condition is $\omega-l \omega_{t r}=0$, with $l$ being integer, and resonant particles satisfying $\left|v_{\|, \text {res }} / v_{t i}\right| \sim O(q / l)$ due to the GAM/EGAM frequency ordering. The subscript "res" denotes resonant particles. Furthermore, the "population" of particles for each transit resonances is proportional to $\left.J_{l}^{2}(\hat{\Lambda}) \partial_{E} F_{0}\right|_{v_{\|, r e s}}$. Noting that $\hat{\Lambda}_{r e s} \sim k_{r} \rho_{i} q^{2} / l$ and the properties of Bessel functions, one can truncate the summation in equation (44) at finite $l$ [12, 13] in the small drift orbit limit with $k_{r} \rho_{i} q^{2} \ll 1$. GAM collisionless damping due to the primary transit resonance $\left(|\omega|=\left|\omega_{t r}\right|\right)$ only was investigated in Ref. 12. It was shown by Sugama et al [13] that, for increasing 
$k_{r} \rho_{t i} q^{2}$, GAM collisionless damping can be significantly enhanced by the increasing weight of higher order transit resonances due to the finite orbit width effect; and the analytical expression including $|\omega|=2\left|\omega_{t r}\right|$ resonance was derived. By further increasing $\hat{\Lambda}_{r e s}$ due to larger $k_{r}$ or $q$, however, more and more transit resonances are needed for the accurate description of GAM collisionless damping [14], and the analytical expression is very difficult to obtain due to the non-trivial task of summing up all the transit resonances.

An alternative approach was developed in Ref. [3], to derive the analytical expression of GAM collisionless damping rate in the short wavelength limit $\left(k_{r} \rho_{i} q^{2} \gg\right.$ $1)$, with all the transit resonances taken into account. Here, we will first show that, the perturbed distribution function for resonant particles derived in Refs. 3, 4] are equivalent to the general solution of equations (3) or (4) in the proper limit, and then briefly summarize the main idea of this approach [3]; while interested readers may refer to Ref. [4] for the detailed derivation.

In the large orbit limit, equation (31) can be expanded using the smallness parameter $1 / \dot{\psi}$, with $|\dot{\psi}| \sim$ $\left|\hat{\omega}_{d} / \omega_{t r}\right| \gg 1$ in the large orbit limit and having denoted derivation of $\psi(\theta)$ with respect to $\theta$ as $\dot{\psi}$ for brevity. Noting that

$$
\begin{aligned}
\int_{-\infty}^{\theta} e^{\psi\left(\theta^{\prime}\right)} d \theta^{\prime} & =\frac{e^{\psi}}{\dot{\psi}}-\frac{e^{\psi}}{\dot{\psi}} \frac{\partial}{\partial \theta} \frac{1}{\dot{\psi}}+\frac{e^{\psi}}{\dot{\psi}} \frac{\partial}{\partial \theta}\left(\frac{1}{\dot{\psi}} \frac{\partial}{\partial \theta} \frac{1}{\dot{\psi}}\right) \\
& -\int_{-\infty}^{\theta} e^{\psi\left(\theta^{\prime}\right)} \frac{\partial}{\partial \theta^{\prime}}\left(\frac{1}{\dot{\psi}} \frac{\partial}{\partial \theta^{\prime}}\left(\frac{1}{\dot{\psi}} \frac{\partial}{\partial \theta^{\prime}} \frac{1}{\dot{\psi}}\right)\right) d \theta^{\prime}
\end{aligned}
$$

one then has

$$
\begin{aligned}
\delta H_{s}=\frac{\omega}{\omega_{t r}} \hat{S}\left[\frac{1}{\dot{\psi}}-\frac{1}{2} \frac{\partial}{\partial \theta}\left(\frac{1}{\dot{\psi}}\right)^{2}+\right. & \frac{1}{2 \dot{\psi}} \frac{\partial^{2}}{\partial \theta^{2}}\left(\frac{1}{\dot{\psi}}\right)^{2} \\
& \left.+O\left(\dot{\psi}^{(-4)}\right)\right] .
\end{aligned}
$$

Noting that $\dot{\psi}=-i\left(\omega-\hat{\omega}_{d} \sin \theta\right) / \omega_{t r}$, the three terms in the square bracket of equation (5) corresponds, respectively, to $\delta H_{\text {res }}^{(0)}, \delta H_{\text {res }}^{(1)}$ and $\delta H_{\text {res }}^{(2)}$ in equations (16), (21) and (23) of Ref. [4], in the $T_{e} / T_{i} \ll 1$ limit assumed here. Thus, the $\delta H_{\text {res }}$ 's in Ref. [4] are equivalent to the general solution of equation (4) by summing up all the transit harmonics, and the underlying wave-paricle interactions in the short wavelength limit are indeed through transit resonances, as pointed out in Ref. [4]. The first term in equation (5) corresponds to the perturbed resonant particle distribution function in the $q \rightarrow \infty$ limit; the third term gives the $O\left(1 / q^{2}\right)$ corrections while the second term vanishes in the surface average.

Since we are interested in the collisionless damping due to thermal ion contribution, a single thermal ion species with Maxwellian distribution function can be assumed, and the GAM dielectric function is derived from the surface averaged quasi-neutrality condition

$$
D_{G} \equiv\left\langle-\frac{e}{T_{i}} F_{0} \overline{\delta \phi}_{G}+J_{G} \overline{\delta H_{i}}\right\rangle /\left(\frac{e}{T_{i}} n_{0} \overline{\delta \phi}_{G}\right) .
$$

The imaginary part of $D_{G}$ due to resonant particle contribution, to the leading order, is then

$$
D_{i}^{(0)}=\operatorname{Im}\left\langle\frac{F_{0}}{n_{0}} J_{G}^{2} \omega \int \frac{d \theta}{2 \pi} \frac{1}{\omega-\omega_{d}}\right\rangle .
$$

We note that, even though in equation (6) the antiHerimitian part comes from the imaginary part of $1 /(\omega-$ $\left.\omega_{d}\right)$, the underlying interaction is not a "drift resonance" [2], since $\omega_{d} \propto \sin \theta$ is temporally fast varying and the effective energy exchange is due to transit resonances as shown in equation (4). The surface average is then carried out by expanding $\omega_{d}$ round $\theta= \pm \pi / 2$ where $\left|\omega_{d}\right|$ is maximized and the integration in $\theta$ is performed by the method of steepest descent. Again, readers interested in the details of the algebra can consult Ref. [4. Here, we will briefly summarize the main ideas underlying the derivation:

1 considering the wave-particle interaction on the time scale of $\left|\omega_{d}\right|^{-1}$, which is much shorter than the transit time $\left|\omega_{t r}\right|^{-1}$ in the large orbit limit, corresponds to the inclusion of a broad spectrum in frequency, i.e., all the transit harmonics are taken into account;

2 for resonant particles, the dominant energy exchange with GAM is captured noting that the waveparticle energy exchange is caused by the acceleration in the radial direction associated with the radial magnetic drift, i.e., $\dot{E}=(e / m) \mathbf{V}_{d} \cdot \delta \mathbf{E}_{r}$, which maximises around $|\theta|=\pi / 2$. Here, $V_{d} \equiv$ $\left(v_{\perp}^{2} / 2+v_{\|}^{2}\right) \sin \theta \mathbf{e}_{r} /\left(\Omega_{i} R_{0}\right)$ is the radial component of magnetic drift velocity.

3 Noting again that $\omega_{d} \propto \sin \theta$ is maximized around $|\theta|=\pi / 2$, ions with lower energy and thus, proportionally (exponentially for a Maxwellian distribution with typical parameters) larger population, will contribute to the resonance.

As a further application, EGAM excitation by EPs in the large magnetic drift orbit limit will be investigated; which is part of the motivation of this communication. To focus on the wave-particle resonance in the short wavelength limit considering the effect of finite magnetic drift orbit averaging, we take $T_{e} / T_{i} \ll 1$ and further neglect the finite Larmor radius effect of EPs. Thus, the leading order EP response to GAM can be derived as

$$
\delta H_{h}=-\frac{e}{m} \partial_{E} F_{0 h} \overline{\delta \phi}_{G} \frac{\omega}{\omega-\omega_{d}},
$$

and the linear dispersion relation of EGAM can be obtained from the quasi-neutrality condition

$$
\hat{\mathscr{E}}_{E G A M} \equiv\left(\overline{\delta n_{i}}+\overline{\delta n_{h}}\right) /\left(e n_{0} \overline{\delta \phi}_{G} / T_{i}\right) .
$$

As the expression of thermal ion density perturbation can be found in Ref. [3, we will focus on the EP density 
perturbation,

$$
\overline{\delta n_{h}}=-\frac{e}{m} B_{0} \sum_{\sigma= \pm 1} \int \frac{E d E d \Lambda}{\left|v_{\|}\right|} \int d \theta \frac{\partial F_{0 h}}{\partial E} \overline{\delta \phi}_{G} \frac{\omega_{d}}{\omega-\omega_{d}} .
$$

Here, $\Lambda=\mu / E$ is the usual definition of the particle pitch angle in velocity space, with $\mu=v_{\perp}^{2} /(2 B)$ the magnetic moment. Noting that $\omega_{d}=\hat{\omega}_{d} \sin \theta$ maximizes at $\theta \simeq$ $\pi / 2$, the contribution around $\theta \simeq \pm \pi / 2$ dominates where wave-particle power exchange maximizes. Taking $x=$ $\theta-\operatorname{sign}(\theta) \pi / 2$, one then has

$$
\begin{aligned}
\int d \theta \frac{\omega_{d}}{\omega-\omega_{d}} & =-2 \pi+\omega \int_{-\infty}^{\infty} d x \frac{1}{\omega-\hat{\omega}_{d}\left(1-x^{2} / 2\right)} \\
& =-2 \pi \frac{i}{\sqrt{\left(2 \hat{\omega}_{d} / \omega\right)\left(\hat{\omega}_{d} / \omega-1\right)}} .
\end{aligned}
$$

In equation (7), the contribution of non-resonant adiabatic particle response is neglected, and the perturbed EP density is then

$$
\begin{aligned}
\overline{\delta n_{h}} & =2 \pi i B_{0} \frac{e}{m}{\overline{\delta \phi_{G}}} \\
& \times \sum_{\sigma= \pm 1} \int \frac{E d E d \Lambda}{\left|v_{\|}\right|} \frac{\partial_{E} F_{0 h}}{\sqrt{\left(2 \hat{\omega}_{d} / \omega\right)\left(\hat{\omega}_{d} / \omega-1\right)}} .
\end{aligned}
$$

Taking a single-pitch angle slowing down EP distribution function [9] as that for neutral beam injection, i.e., $F_{0 h}=c_{0} \delta\left(\Lambda-\Lambda_{0}\right) H_{E}$, with $c_{0}=$ $n_{b} \sqrt{2\left(1-\Lambda_{0} B_{0}\right)} /\left(4 \pi B_{0} \ln \left(E_{b} / E_{c}\right)\right), n_{b}$ is the density of the EP beam, $E_{b}$ and $E_{c}$ being respectively the EP birth and critical energies, $\delta(x)$ is the Dirac delta function, and $H_{E}=1 /\left(E^{3 / 2}+E_{c}^{3 / 2}\right) \Theta\left(1-E / E_{b}\right)$ with $\Theta\left(1-E / E_{b}\right)$ being the Heaviside step function. The integration in velocity space can then be carried out, and yields the short wavelength EGAM dispersion relation:

$$
\begin{array}{r}
\hat{b}_{i}\left(-1+\frac{\omega_{G}^{2}}{\omega^{2}}\right)+\Delta_{f}+i n_{b}\left[\frac{-2+3 \Lambda_{0} B_{0}}{1-\Lambda_{0} B_{0}} \frac{\Omega_{b}}{\omega} \sqrt{\frac{\Omega_{b}}{\omega}-1}\right. \\
\left.-\Lambda_{0} B_{0} \frac{\left(\omega / \Omega_{b}\right)^{1 / 2}}{\sqrt{\Omega_{b} / \omega-1}}\right]=0
\end{array}
$$

with $\Delta_{f}$ being the non-resonant EP contribution

$\Delta_{f}=n_{b}\left[\frac{-2+3 \Lambda_{0} B_{0}}{1-\Lambda_{0} B} \frac{\Omega_{b}}{\omega}+\Lambda_{0} B_{0}\left(\frac{\omega}{\Omega_{b}}\right)^{1 / 2}\left(\frac{E_{b}}{E_{c}}\right)^{3 / 2}\right]$,

$\Omega_{b} \equiv \hat{\omega}_{d}\left(E=E_{b}\right), \quad \hat{b}_{i}=k_{r}^{2} \rho_{t i}^{2} / 2$, and $\omega_{G} \simeq$ $\sqrt{7 / 4+\tau} v_{t i} / R_{0}$ is the GAM frequency.

The first term in the square bracket of equation (9) $(\propto$ $\sqrt{\Omega_{b} / \omega-1}$ ) could be the destabilizing term depending on the value of $\Lambda_{0} B_{0}$, while the second term $\left(\propto\left(\Omega_{b} / \omega-\right.\right.$ $1)^{-1 / 2}$ ) is stabilizing. As a result, EGAM excitation in the large orbit limit requires, first,

$$
\Lambda_{0} B_{0}>2 / 3
$$

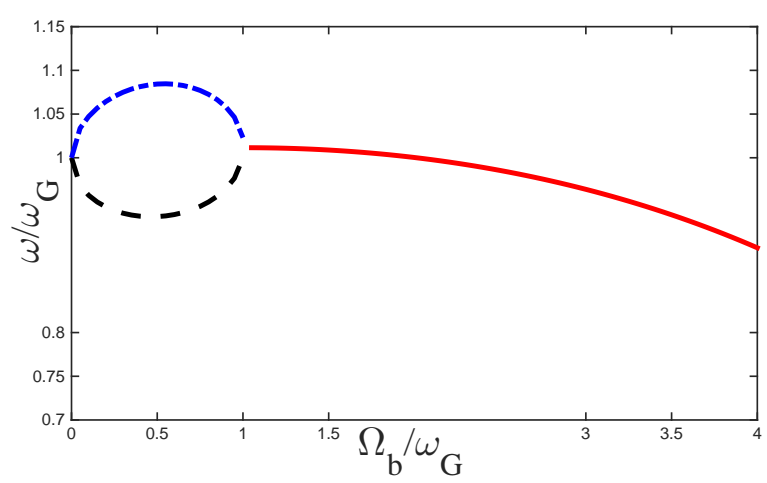

FIG. 1: Real frequency v.s. $\Omega_{b} / \omega_{G}$

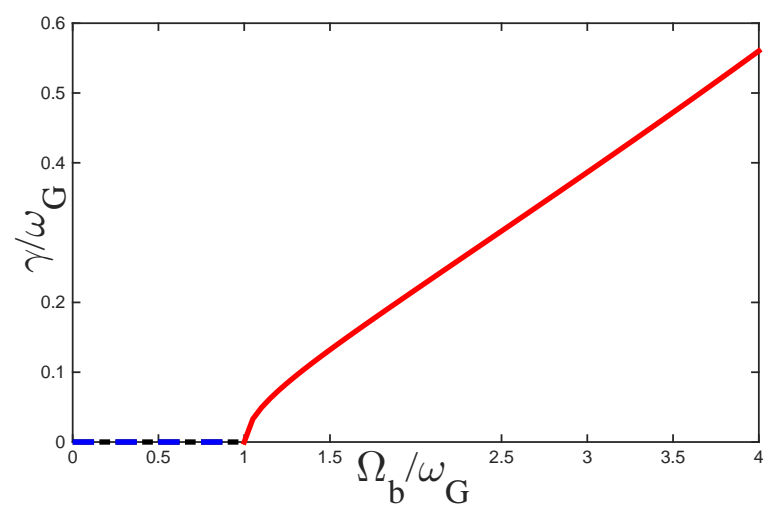

FIG. 2: Growth rate v.s. $\Omega_{b} / \omega_{G}$

for the first term of EP contribution in equation (91) to be destabilizing; and second, $\Omega_{b} / \omega$ being sufficiently large for the short wavelength EGAM to be unstable.

The dispersion relation is solved numerically as a function of $\Omega_{b} / \omega_{G}$. Note that from our previous analysis, the drive due to wave-particle resonance exists only for $\left|\Omega_{b} / \omega\right|>1$; and that the destabilizing term increases with $\left|\Omega_{b} / \omega\right|$ while the stabilizing term decreases with $\left|\Omega_{b} / \omega\right|$, so the destabilizing term is neglected, which gives negligible contribution for $\left|\Omega_{b} / \omega\right|>1$ where waveparticle energy exchange exists. The other parameters are taken as follows: $n_{b} / \hat{b}_{i}=0.3, \Delta_{f}=0$, and the obtained short wavelength EGAM real frequency and growth rate dependences on $\Omega_{b} / \omega_{G}$ are shown in Figs. 1 and 2 respectively. It is shown that, the EGAM real frequency decreases slightly with increasing $\Omega_{b} / \omega_{G}$, and the unstable EGAM frequency is always smaller than local GAM frequency. On the other hand, as the EGAM is unstable for $\Omega_{b} / \omega_{G}>1$, the growth rate increases with $\Omega_{b}$. For $\Omega_{b} / \omega_{G}$ significantly larger than unity, the growth rate increases almost linearly with $\Omega_{b} / \omega_{G}$, and thus, $E_{b}$, as is clearly seen from the destabilizing term of equation (91). This is due to the increasingly dense high order transit resonances associated with increasing $E_{b}$. Whereas, in 
the long wavelength limit, the growth rate will be peaked when the EP parallel velocity at birth energy satisfies a certain harmonic resonance, similar to the case for GAM Landau damping discussed in Ref. [13].

In conclusion, the underlying physics picture of waveparticle resonances at short wavelength is clarified, taking short wavelength GAM collisionless damping as an example. Assuming large aspect ratio tokamak and well circulating particles, the ion response to GAM is derived from linear gyrokinetic equation by integration along unperturbed guiding-center orbit. The general solution is then obtained by expansion into transit harmonics, with the "population" of resonant particles to each transit harmonic proportional to $\left.J_{l}^{2}\left(\hat{\Lambda}_{r e s}\right) \partial_{E} F_{0}\right|_{v_{\|, \text {res }}}$. As a result, to obtain the GAM collisionless damping in the short wavelength limit, all the transit resonances must be kept. It is then shown that, the result obtained in Ref. 3 based on large orbit width expansion, is equivalent to the general solution up to $O\left(1 /\left(k_{r} \rho_{t i} q^{2}\right)\right)$; and the underlying physics for wave-particle interactions at short wavelength consists indeed in the summation of all the transit resonances.
As a further application, the EGAM excitation at short wavelengths is also investigated, and the analytical dispersion relation is derived assuming a single pitch angle slowing down EP distribution function. Our results indicates that the short wavelength EGAM dispersion relation depends algebraically on the EP characteristic frequency, instead of the logarithmic dependence characterizing the long wavelength limit, which is typical for a slowing down EP distribution function. The short wavelength EGAM is unstable for $\Omega_{b}>\omega_{G}$, and $\Lambda_{0} B_{0}>2 / 3$. For $\Omega_{b}$ significantly larger than GAM frequency, the short wavelength EGAM growth rate is proportional to $\Omega_{b}$, and thus, EP birth energy $E_{b}$ due to the increasingly denser high order transit resonances as $\Omega_{b} \gg \omega_{G}$.

This work is supported by US DoE GRANT, the National Magnet Confinement Fusion Research Program under Grants Nos. 2013GB104004 and 2013GB111004, the National Science Foundation of China under grant Nos. 11575157 and 11235009, Fundamental Research Fund for Chinese Central Universities under Grant No. 2017FZA3004 and EUROfusion Consortium under grant agreement No. 633053.
[1] N. Winsor, J. L. Johnson, and J. M. Dawson, Physics of Fluids 11, 2448 (1968).

[2] M. Sasaki, N. Kasuya, K. Itoh, K. Hallatschek, M. Lesur, Y. Kosuga, and S.-I. Itoh, Physics of Plasmas 23, 102501 (2016).

[3] F. Zonca and L. Chen, Europhys. Lett. 83, 35001 (2008).

[4] Z. Qiu, L. Chen, and F. Zonca, Plasma Physics and Controlled Fusion 51, 012001 (2009).

[5] W. Zhang, Z. Lin, L. Chen, Physical review letters 101, 095001 (2008).

[6] Z. Feng, Z. Qiu, and Z. Sheng, Physics of Plasmas (1994present) 20, 122309 (2013).

[7] R. Nazikian, G. Fu, M. Austin, H. Berk, R. Budny, N. Gorelenkov, W. Heidbrink, C. Holcomb , G. Kramer, G. McKee, M. Makowski, W. Solomon, M. Shafer,
E. Strait, and M. Van Zeeland, Phys. Rev. Lett. 101, 185001 (2008).

[8] G. Fu, Phys. Rev. Lett. 101, 185002 (2008).

[9] Z. Qiu, F. Zonca, and L. Chen, Plasma Phys. Control. Fusion 52 (2010).

[10] P. Rutherford and E. Frieman, Physics of Fluids 11, 569 (1968).

[11] J. Taylor and R. Hastie, Plasma Physics 10, 479 (1968).

[12] F. Hinton and R. M., Plasma Physics and Controlled Fusion 41, A653 (1999).

[13] H. Sugama and T.-H. Watanabe, Journal of plasma physics 72, 825 (2006).

[14] X. Xu, Z. Xiong, Z. Gao, W. Nevins, and G. McKee, Physical review letters 100, 215001 (2008). 